\title{
Analysis of Dynamic Mechanical Properties for Simply Supported Beam Including ESP Glass
}

\author{
Guoliang Zhang and Jishi Du \\ Institute of Electronic Engineering, China Academy of Engineering Physics, Mianyang, Sichuan, China
}

\begin{abstract}
There is growing awareness of the need to protect system circuit from the abnormal impact loading environment. Impact mechanical sensor can immediately hold back system circuit in the extreme environment. Especially, mechanical response component of it must possess the characteristics of high fracture strength and high Weibull modulus simultaneously. Engineered stress profiles glass (ESP glass) is the best choice for achieving these two performances in all kinds of material. In many cases, it is desirable that once the impact mechanical sensor has served its function that ESP glass then fractures into very small fragments. ESP glass with controlled static fracture properties so far has been carried out theoretically and experimentally, whereas impact properties of it are rarely performed. In order to understand mechanical properties of ESP glass under impact loading environment, the mechanical behavior of the simply supported beam including ESP glass is investigated by theoretical formulae and finite element method (FEM) simulation in this paper. The theoretical computational results of the dynamic flexural strength of ESP glass are consistent with the simulation results of it. Results gained show that the relation between static mechanical properties and dynamic mechanical properties of ESP glass can be established by dynamic coefficient.
\end{abstract}

\section{Introduction}

With growing awareness of the need to protect system circuit from the abnormal impact loading environment, mechanical response properties of impact mechanical sensor have been a topic of growing interest gradually [1], [2]. As the key part, mechanical response component of the sensor must possess the characteristics of high fracture strength and high Weibull modulus simultaneously. With controlled fracture properties, ESP glass has very high reliability strength of four to five times that of regular glass. At same time, visible warning cracks appeared on the surface of ESP glass are generated before disastrous failure occurs. At this level, the advantages of a Weibull modulus are shown in Fig. 1. It assume that the ESP glass must sustain $80 \%$ of the average failure stress. At a given stress is increased significantly with higher Weibull modulus of 60 , only a one in one million chance of failing will occur for this ESP glass. In contrast, regular annealed glass with lower Weibull modulus of 5 has a $30 \%$ failure probability [3]. Although regular glass does not have a microstructure, ESP glass has been designed a built-in stress profile that would produce R-curve (increasing crack resistance) behavior. Based on this design idea, there is a high compressive stress just below the glass surface rather than at the surface (Fig. 2). This devise can also make glass strength controlled by the size of the worst defect.
Therefore, ESP glass is the best choice for achieving these two performances in all kinds of material. In many cases, it is desirable that once the impact mechanical sensor has served its function that ESP glass then fractures into very small fragments [4]. Under impact loading environment, it will interfere with the functioning of system circuit of a device drastically. ESP glass with controlled static fracture properties so far has been carried out theoretically and experimentally [5]-[7], whereas impact properties of it are rarely performed. Meanwhile, analysis of dynamic load and dynamic coefficient of regular elastic and plastic material had been researched many years [8]-[10]. Although the material properties of ESP glass vary considerably from regular elastic and plastic material, these investigations can be referred to studying impact properties of ESP glass.

In order to understand mechanical properties of ESP glass under impact loading environment, the mechanical behavior of the simply supported beam including ESP glass is investigated by theoretical formulae and finite element method (FEM) simulation in this paper. Hence, theoretical formulae and FEM simulation are simultaneously used to know the relation between static mechanical properties and dynamic mechanical properties of ESP glass. It also can be used to understand mechanical properties of ESP glass under impact loading environment. Moreover, the results gained can be utilized to improve the structure design of impact mechanical 
sensor and adequately exert advantages of ESP glass with controlled fracture properties.

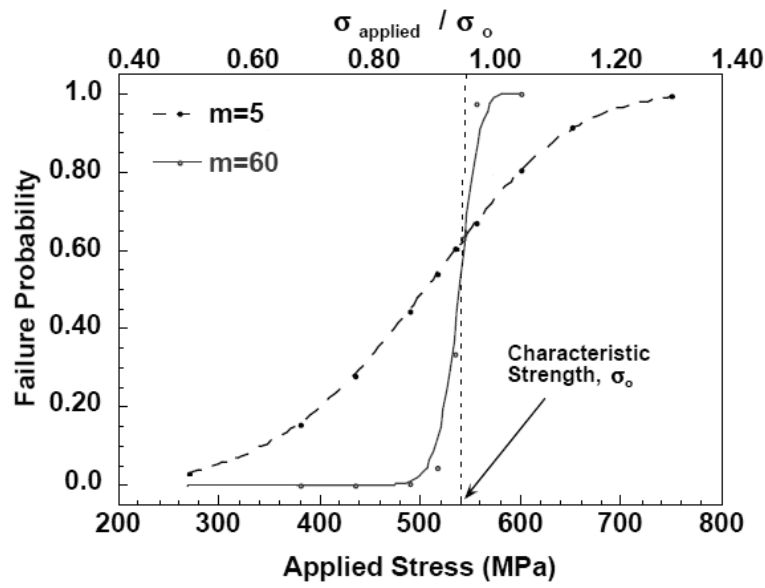

Figure 1. The relationship between failure probability and various Weibull moduli (m).

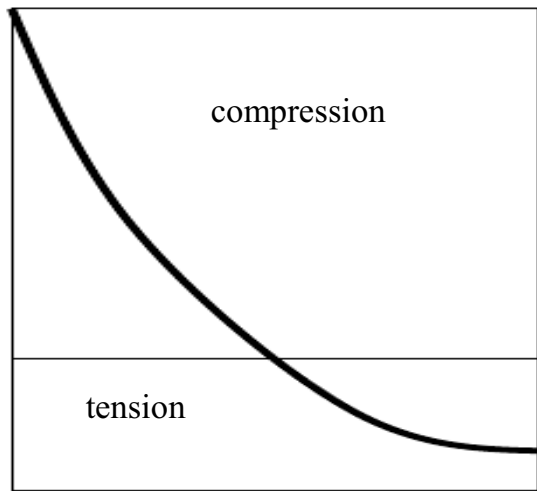

(a)

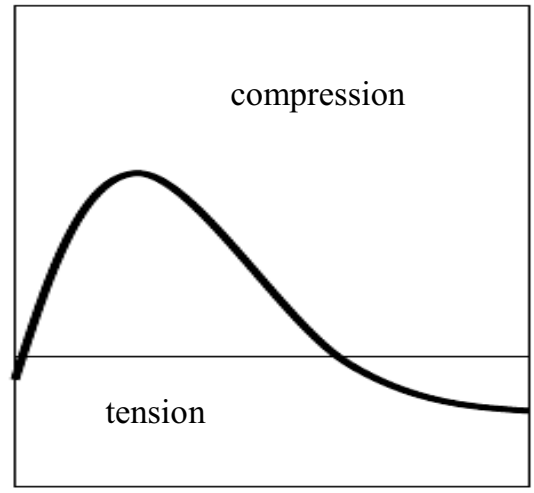

(b)

Figure 2. Stress profiles of two kinds of glass: (a) regular annealed glass, (b) ESP glass.

\section{Theoretical calculation of a dynamic stress in a simply supported beam}

A simply supported beam is loaded with different types of force in Fig. 3. Theoretical calculation of a dynamic stress in this beam can be acquired from the energy equilibrium condition [11]. Kinematics energy of a mass of block is equal to a work done during a dislocation with dynamic deformation and free falling height. The mass of block $\mathrm{C}$ is $m$. The length of beam is L. In Fig. 3(a), a static force $F_{j}=\mathrm{mg}$ is acted on the middle of the beam whose bending deformation is calculated from the equation (1) as follows:

$$
t_{j}=\frac{F_{j} L^{3}}{48 E I_{z}}
$$

where $t_{j}$ indicates the static deformation, $\mathrm{E}$ is the elastic modulus, $I_{z}$ is the cross-section moment of inertia for $Z$ axis, $E I_{z}$ is the bending stiffness and its value is constant. In Fig. 3(b), the dynamic force $F_{d}$ will be generated as the block $\mathrm{C}$ is falling from the height $h$ and impacting on the middle of the beam. Its bending deformation is calculated from the equation (2) as follows:

$$
t_{d}=\frac{F_{d} L^{3}}{48 E I_{z}}
$$

where $t_{d}$ indicates the dynamic deformation whose value is larger than the static deformation $t_{j}$.

Combined with equation (1) and equation (2), the dynamic force $F_{d}$ is given as:

$$
F_{d}=\frac{t_{d}}{t_{j}} F_{j}
$$

in which the ratio of the dynamic deformation $t_{d}$ to the static deformation $t_{j}$ denotes the dynamic coefficient $K_{d}$. It is expressed as:

$$
K_{d}=\frac{t_{d}}{t_{j}}
$$

The dynamic coefficient $K_{d}$ can be calculated from the energy equilibrium condition which is read as follows:

$$
F_{j}\left(h+t_{d}\right)=\frac{1}{2} F_{d} t_{d}
$$

Combined with equation (3) and equation (5), the dynamic coefficient $K_{d}$ becomes:

$$
K_{d}=1+\sqrt{1+\frac{2 h}{t_{j}}}
$$

The free falling height $h$ can be deduced from the energy equilibrium condition as follows:

$$
h=\frac{(a t)^{2}}{2 g}
$$

where $a$ is impact acceleration, $g$ is gravity acceleration, $t$ is impact time.

The static flexural strength of brittle materials is given as:

$$
\sigma_{j}=\frac{8 F_{j} L}{\pi d^{3}}
$$

where $d$ is diameter of the beam. Associated with equation $(1) \sim(7)$, the dynamic flexural strength $\sigma_{d}$ of ESP glass within the simply supported beam could be reckoned. 


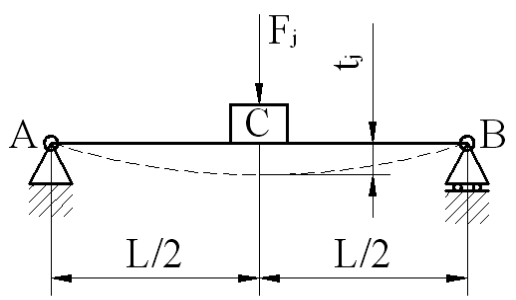

(a)

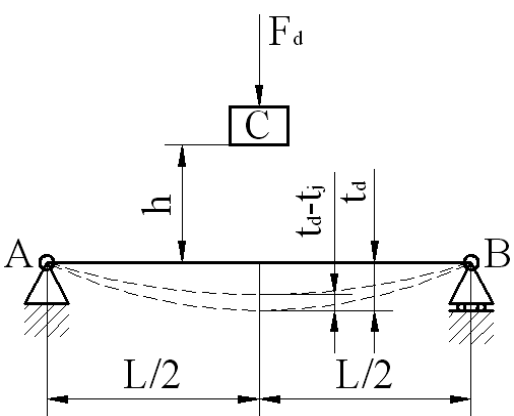

(b)

Figure 3. Schematic of a simply supported beam with different types of force: (a) static force, (b) dynamic force.

\section{Finite element analysis}

Under impact loading environment, the dynamic stress on ESP glass will be greater than the fracture strength on it. For validating theoretical calculation of a dynamic stress in a simply supported beam including ESP glass, a threedimensional finite element model of it is established in ANSYS simulator (Fig. 4). Non-uniform grid is introduced for reducing the amount of meshing as well as receiving the dynamic stress distributions in the middle concerned region. Hexahedron grids are introduced in the entire computational region for enhancing the accuracy of simulation.

In Fig. 4, the beam is ESP glass, the mass of block is stainless steel, the foundation is stainless steel, and the support post is stainless steel. The geometric and mechanic characteristics of main components are summarized in Table 1. The condition of first row is for the beam. The condition of second row is for mass of block. Thereinto, $L$ is the length, $D$ is the diameter, $E$ is elastic modulus, $v$ is Poisson's ratio, $\rho$ is density, $\sigma_{f}=400 \mathrm{MPa}$ is fracture strength of ESP glass, $a$ is impact acceleration, $t$ is impact time.

The assumptions for computational region of the simply supported beam are given as follows: 1) The mass of block, the foundation and the support post are set as rigid body; 2) The beam, the foundation and the support post are tied up together; 3 ) The friction generated on the dynamic contact surfaces is ignored.

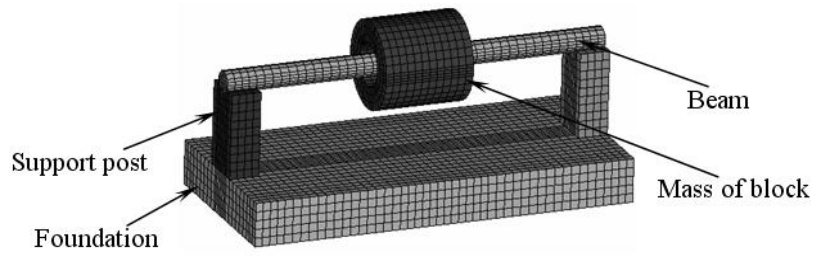

Figure 4. Proposed finite element composite model.

\section{Results and discussions}

\subsection{Theoretical calculation}

Be based upon Table 1 and equation (7), the free falling height $h$ is about $31 \mathrm{~m}$. From equation (1), the static bending deformation $t_{j}$ is $9.8 \mathrm{E}-5 \mathrm{~mm}$ and the static force $F_{j}$ is $0.013 \mathrm{~N}$. Then, according to equation (3) and equation (4), the dynamic coefficient $K_{d}$ is near 4E3 and the dynamic force $F_{d}$ is close to $52 \mathrm{~N}$. Therefore, in the light of the dynamic flexural strength of brittle materials, the dynamic flexural strength $\sigma_{d}$ of ESP glass is about $415 \mathrm{MPa}$. It can be seen that the dynamic stress on the ESP glass has been greater than the fracture strength on it under the given impact loadings from Table 1.

Table 1. Geometric and mechanic characteristics of simply supported beam and initial conditions.

\begin{tabular}{|c|c|c|c|c|c|c|}
\hline $\begin{array}{l}L \\
(\mathrm{~mm})\end{array}$ & $\begin{array}{l}D \\
(\mathrm{~mm})\end{array}$ & $\begin{array}{l}\rho \\
\left(\mathrm{T} / \mathrm{mm}^{3}\right)\end{array}$ & $\begin{array}{l}E \\
(\mathrm{MPa})\end{array}$ & $v$ & $\begin{array}{l}a \\
\left(\mathrm{~m} / \mathrm{s}^{2}\right)\end{array}$ & $\begin{array}{l}t \\
\text { (ms) }\end{array}$ \\
\hline 25 & 2 & $2.2 \mathrm{E}-9$ & $5.5 \mathrm{E} 4$ & 0.25 & 0 & 0 \\
\hline 6 & 6 & $7.8 \mathrm{E}-9$ & $2 \mathrm{E} 5$ & 0.3 & 5E4 & 0.5 \\
\hline
\end{tabular}

On the basis of the above analysis, the dynamic load resulting from an impact load is bigger than a static load loaded by the same mass. In other words, the same mass will cause very different loads on the same beam as this mass falls from 0 to the specifically height.

Properties of all kinds of material are also significant for theoretical analysis. Impulse loads maybe alter the attributes of the material.

\subsection{Simulation}

Fig. 5 shows the response of impact acceleration during the mass of block is freely falling from height $h$ and impacting on the beam. The response of impact acceleration produced on the beam fast changes from the maximum $5 \mathrm{E} 4 \mathrm{~m} / \mathrm{s}^{2}$ to zero. It is consistent with the load conditions from Table 1 . The kinematics energy of the mass of block transforms into a potential energy of an elastic deformation for the beam. Meanwhile, the response of impact acceleration appeared on the foundation fast changes from the maximum $4 \mathrm{E} 4 \mathrm{~m} / \mathrm{s}^{2}$ to zero. It should be noted that the response of impact acceleration will be attenuated as it is transferring from the beam to the foundation. Combined the energy equilibrium condition, the more realistic dynamic stress distributions can be gotten

Fig. 6 depicts dynamic stress contour on the ESP glass as the response of impact acceleration of the beam reaches its maximum. The maximum dynamic stress is located at the middle of the beam with different lengths. As the length of the beam is $25 \mathrm{~mm}$, this maximum dynamic stress of $428 \mathrm{MPa}$ agrees well with the theoretical computational results of it. When the length of the beam becomes $13 \mathrm{~mm}$, the maximum dynamic stress will increase up to $624 \mathrm{MPa}$ under the greater response of impact acceleration of $8 \mathrm{E} 4 \mathrm{~m} / \mathrm{s}^{2}$. In summarized, ESP glass has fractured under the given impact load conditions. 


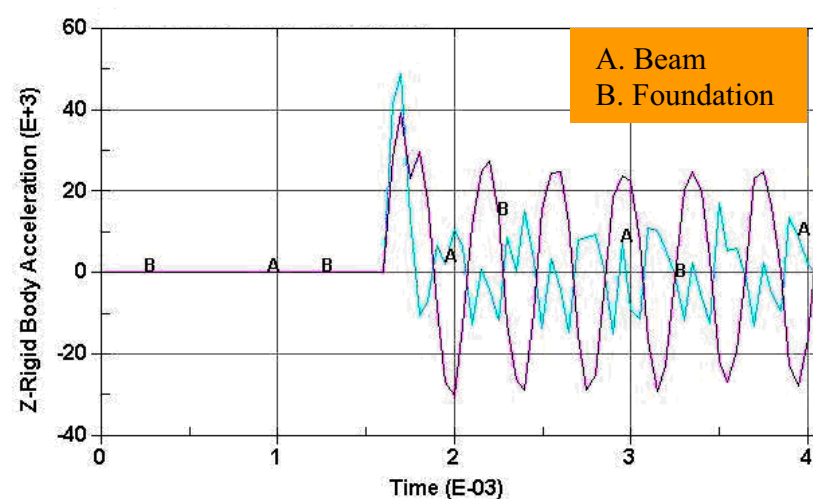

Figure 5. Response of impact acceleration.

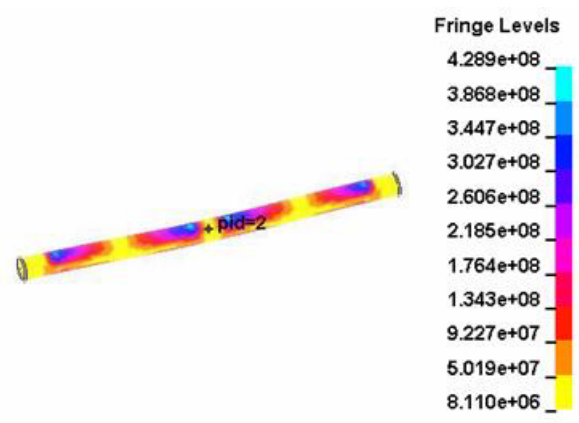

(a)

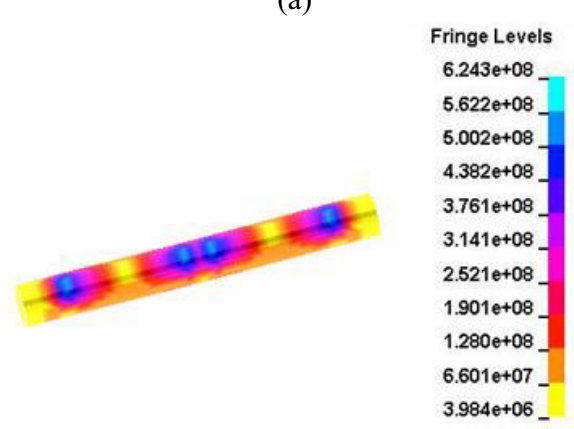

(b)

Figure 6. Dynamic stress contour on the ESP glass with different length: (a) $L$ of the beam is $25 \mathrm{~mm}$, (b) $L$ of the beam is $13 \mathrm{~mm}$.

\section{Conclusions}

Impact mechanical sensor has been a topic of growing interest with the growing awareness of the need to protect system circuit from the abnormal impact loading environment. As the key part of the impact mechanical sensor, ESP glass is the best choice for achieving these two performances of the characteristics of high fracture strength and high Weibull modulus in all kinds of material.

In order to understand mechanical properties of ESP glass under impact loading environment, the mechanical behavior of the simply supported beam including ESP glass is investigated by theoretical formulae and FEM simulation in this paper. Moreover, the relation between static mechanical properties and dynamic mechanical properties of ESP glass has been established by dynamic coefficient. Based on the energy equilibrium condition, the better agreement of the dynamic stress produced on the ESP glass between theoretical calculation and simulation has been obtained. These results show that research of the dynamic impulse for an impact mechanical sensor probably discovers the value of the dynamic stress as well as a dynamic coefficient. It is notable that impact loads may radically change the mechanical properties of the material. For improving the structure design, calculated protection coefficients for an impact mechanical sensor containing a bending beam should be higher than the ones used during designing. ESP glass and stainless steel mentioned in this article should be assumed as the introduction to the further theoretical analysis as well as practical verification.

\section{Acknowledgement}

The authors are very grateful to our colleagues for their discussion and data processing. This work is supported financially by the Science and Technique Innovation Foundation of Institute of Electronic Engineering of China Academy of Engineering Physics, China (Grant No. S20140806).

\section{References}

1. B. Denkena, F.L. Hackeloer, 2010 IEEE Sensors 2518-2524 (2010)

2. A. Stevan, 2012 Mediterranean Conference on Embedded Computing 50-53 (2012)

3. D.J. Green, R. Tandon, V.M. Sglavo, Science. 283, 1295 (1999)

4. D.J. Green, J Non-Cryst Solids. 316, 35 (2003)

5. V.M. Sglavo, A. Prezzi, D.J. Green, Eng Fract Mech. 74, 1383 (2007)

6. S. Blanka, S. Jarmila, N. Pavla, J Non-Cryst Solids. 356, 1509 (2010)

7. P.K. Kreski, A.K. Varshneya, A.N Cormack, J NonCryst Solids. 358, 3539 (2012)

8. P. Ratko, K. Predrag, R. Predrag, Journal of Sound and Vibration. 301, 690 (2007)

9. E. Wlodarczyk, M. Zielenkiewicz, Journal of Theoretical and Applied Mechanics. 49, 457 (2011)

10. B. Rosnawati, M.R. Munzilah, E.A. Mohd, Applied Mechanics and Materials. 405, 1900 (2013)

11. Z.X. Gong, Z.Z. Li, Materials and Mechanics, Science Press, Beijing, (1999) (in Chinese) 